084

NEUROLOGICAL PRESENTATIONS OF RARE CHLOROMAS IN THE CONTEXT OF HAEMATOLOGICAL MALIGNANCY

1,25ophie Chatterton, 'Jacob Helou, ${ }^{3}$ James Drummond, ${ }^{4,5}$ Christopher Ward, 4,5 Luke Coyle, $1,5 \mathrm{Karl} \mathrm{Ng} .{ }^{1}$ Department of Neurology, Royal North Shore Hospital, Sydney, NSW, Australia; ${ }^{2}$ University of New South Wales, Sydney, NSW, Australia; ${ }^{3}$ Radiology Department, Royal North Shore Hospital, Sydney, NSW, Australia; ${ }^{4}$ Haematology Department, Royal North Shore Hospital, Sydney, NSW, Australia; ${ }^{5}$ University of Sydney, Sydney, NSW, Australia

\subsection{6/bmjno-2021-ANZAN.84}

Objective(s) To describe the spectrum of neurological presentations of chloromas.

Method Two case reports.

Results A 72 year-old female presented with a right-sided cavernous sinus syndrome on a background of a myeloproliferative disorder. MRI brain demonstrated a FLAIR hyperintense lesion centred on the right cavernous sinus. Cerebrospinal fluid (CSF) was acellular with negative cytology and flow cytometry. FDG PET/CT demonstrated diffuse marrow hypermetabolism, hepatosplenomegaly and focal FDG uptake in the cavernous sinus and a remote paraspinal region, suspicious for chloromas. The patient developed pancytopaenia with blasts, and tumour lysis syndrome (TLS) consistent with transformation to acute myeloid leukaemia (AML). The patient was given pulse methylprednisolone, hydroxyurea and external beam radiotherapy to the cavernous sinus lesion with symptomatic improvement. The second case is of a 76 year-old male who presented with progressive lower limb weakness with acute faecal incontinence. This was on a background of AML treated with induction STIMULus (cytarabine and thioguanine) followed by remission confirmed on a recent bone marrow biopsy. CSF showed a marked lymphocytosis with many undifferentiated cells, and flow cytometry was consistent with AML. An MRI whole spine demonstrated a solitary avidly enhancing intramedullary T11 cord lesion with associated oedema. In the context of AML and CSF pathology this was thought to reflect an intramedullary chloroma. The patient was treated with external beam radiotherapy, pulse intravenous methylprednisolone and intrathecal chemotherapy with symptomatic improvement.

Conclusion These two cases demonstrate the diversity of neurological clinical presentations of chloromas, an unusual collection of leukaemic cells exerting mass-like effect.

\section{BILATERAL ABDUCENS NERVE PALSIES FOLLOWING BILATERAL MIDDLE MENINGEAL ARTERY EMBOLISATION FOR CHRONIC SUBDURAL HAEMORRHAGE}

${ }^{1}$ Subahari Raviskanthan, ${ }^{1}$ Peter W Mortensen, ${ }^{2} Y_{i}$ J Zhang, $1,3,4,5,6$ Andrew G Lee ${ }^{1}$ Department of Ophthalmology, Blanton Eye Institute, Houston Methodist Hospital, Houston, Texas, US; ${ }^{2}$ Department of Neurosurgery, Houston Methodist Hospital and Weill Cornell Medicine, New York, US; ${ }^{3}$ Department of Ophthalmology, University of Texas Medical Branch, Galveston, Texas, US; ${ }^{4}$ Department of Ophthalmology, University of Texas MD Anderson Cancer Center, Houston, Texas, US; ${ }^{5}$ Texas A and M College of Medicine, Bryan, Texas, US; ${ }^{6}$ Department of Ophthalmology, The University of lowa Hospitals and Clinics, lowa City, lowa, US

\subsection{6/bmjno-2021-ANZAN.85}

Background Middle meningeal artery embolisation (MMAE) has recently been proposed as a treatment for subdural haemorrhage (SDH). MMAE aims to decrease the vascular supply to abnormal membranes produced in response to dural space inflammation in SDH.
Methods/Results We report a case of bilateral abducens nerve palsies following bilateral MMAE. A 37-year-old male with Type 1 diabetes, previous traumatic brain injury, and excessive alcohol intake presented with increasing headaches and falls. Brain imaging revealed bilateral $\mathrm{SDH}$ with minimal midline shift to the right $(2-3 \mathrm{~mm})$. He subsequently underwent bilateral MMAE. Immediately post operatively, he noted new binocular horizontal diplopia, with bilateral abducens nerve palsies clinically. Repeat brain imaging revealed stable SDH with new punctate foci of diffusion restriction bilaterally though none that would explain his abducens nerve palsies. There were no brainstem infarctions and there was no midline shift. 2 month post-operative review showed significant improvement in his bilateral abducens nerve palsies.

Conclusions The vascular supply to the abducens nerve is rich, with multiple external carotid artery and internal carotid artery branches, therefore abducens nerve ischemia is rare. Previous studies with provocative lidocaine testing into the middle meningeal artery for other endovascular procedures showed 3 patients developed transient abducens nerve palsy. There are only two cases of transient diplopia within the MMAE related literature, and it is unclear whether these were $\mathrm{CN}$ VI palsies. To our knowledge this is the first reported case of bilateral abducens nerve palsies post MMAE, possibly from indirect ischemia post embolisation of the middle meningeal artery.

\section{AN UNEXPECTED CAUSE OF OPHTHALMOPLEGIA AND AREFLEXIA: THE PUPILS HAVE IT}

${ }^{1}$ Jessica Qiu, ${ }^{1}$ Albert Vien, ${ }^{1}$ Andrew Hannaford, ${ }^{2}$ Patricia Ferguson, ${ }^{3}$ Mohammad Hamidi, ${ }^{3}$ Amit Vaidya, ${ }^{1}$ Andrew Henderson. 'Department of Neurology, Westmead Hospital, Sydney, NSW, Australia; ${ }^{2}$ Department of Infectious Diseases, Westmead Hospital, Sydney, NSW, Australia; ${ }^{3}$ Department of Intensive Care, Westmead Hospital, Sydney, NSW, Australia

\subsection{6/bmjno-2021-ANZAN.86}

Introduction We describe a case of botulism and its differentiation from acute neurological presentations.

Case A 35 year-old man presented with dysphagia, subjective jaw numbness, dyspnoea and vertigo. He denied alcohol intake but had injected methamphetamines and developed symptoms shortly after. 48 hours post-injection, he was drowsy and developed a complete internal and external ophthalmoplegia, an absent gag reflex with slow tongue movements and facial diplegia with sparing of distal muscle groups. His reflexes were initially present but became absent five hours later. There were no sensory or cerebellar findings. Blood cultures were taken given his history of intravenous drug use.

Neuroimaging did not note the presence of a posterior circulation infarct. A lumbar puncture did not demonstrate cytoalbuminological dissociation, and antibody testing for myasthenia gravis and Guillain Barre Syndrome variants were negative, although he was commenced on empirical IVIg prior to these results. He required intubation on day three of admission due to hypoxia secondary to aspiration but needed minimal ventilatory support.

Neurophysiological findings were consistent with a presynaptic neuromuscular junction disorder. Blood cultures noted clostridium botulinum with detection of clostridium botulinum toxin B. Antitoxin was administered on day four of presentation with slow but gradual improvement in his neurology and he was extubated on day 16. 